on a weekly basis to the medical team, through $(\mathrm{P})$ production, email and discussion at communication meetings. Information given highlights recurrent themes, near misses and the learning points from recent HLIs. In tandem, nursing teams receive similar feedback about incidents relevant to the care that they provide.

Results In this 60-cot unit, 40 to 60 critical incidents are reported each month, demonstrating our culture of openness and education. Since May 2017, Messages of the Week have focused around medication errors (prescribing and administration), inter-specialty communication, discharge planning, documentation and WBITs (wrong blood in tubes.) There is a significant improvement in reported awareness of HLIs at all grades.

Conclusion As we strive to improve the quality of care that we provide for our NICU babies and their families, we must continue to explore novel methods of enhancing education and communication within our teams. With challenges in shift working and high turnover of staff in large neonatal units, disseminating a clinical risk Message of the Week to front-line medical and nursing staff is an effective way to ensure lessons are learnt from adverse events.

\section{G414(P) ALWAYS EVENTS: LESSONS IN PAEDIATRIC CARE}

${ }^{1} \mathrm{CM}$ Mak, ${ }^{2} \mathrm{DWF}$ Fenn, 'SS Shanmugalingam. 'Paediatrics, Royal Free London NHS Foundation Trust, London, UK; ${ }^{2}$ Paediatrics, Whittington Health NHS Trust, London, UK

\subsection{6/archdischild-2018-rcpch.403}

Aims 'Always Events' is a framework of practice that aims to improve patient experience through positive goal-setting in a person- and family-centred approach. Through partnering with patients and their families, the aim is to identify fundamental behaviours that should be performed by the healthcare system for every patient, every time ${ }^{1}$.

The aim of this study was to explore families' experience of their inpatient journey to inform the development of Always Events guidelines with the ultimate goal of enhancing families' experience of care.

Methods We conducted one to one structured parent interviews exploring aspects the parents or their child had experienced while on either the general paediatric or neonatal ward. The interviews and questions were designed to encourage open discussion focusing on the patients' journey in the department from admission to discharge, aimed at identifying practices that could be integrated as Always Events. Responses were transcribed, collated and coded.

Results 23 interviews were conducted: 9 from the general paediatric population and 14 from the neonatal unit. The responses were positive, with all parents reporting a high level of care and appreciating being asked what mattered to them. Themes considered to be key in improving their experience included honest and informed communication (both staff to staff and staff to parent), collaborative and practical understanding of care (parental education, familiarisation of the unit before and during admission, and comprehensive discharge decision making), and the creation of a safe and comfortable environment. Parents on the neonatal unit also cited peer support as highly valuable.

Conclusion This pilot study highlighted the value of engaging families in establishing potential Always Events within the paediatric department. Two benefits emerged: firstly, parents enjoyed providing feedback and found the process rewarding; secondly, it identified valuable experiences relating to everyday care which could be explored further and used to develop fundamental principles. Our next stage is to explore these themes through multi-professional and parental focus groups with the aim of co-designing and implementing Always Events.

\section{REFERENCE}

1. A focus on 'always events'. Improving Patient Care. Institute for Healthcare Improvement, 2014.

\section{G415(P) OBSERVATIONS IN A PAEDIATRIC EMERGENCY DEPARTMENT: IMPACT OF SKILLMIX ON TIME SPENT DURING PATIENT ASSESSMENT}

${ }^{1} \mathrm{~A}$ Fuller, ${ }^{2} \mathrm{AF}$ Mellon. 'MBBS Programme, University of Newcastle Medical School, Newcastle upon Tyne, UK; ${ }^{2}$ Paediatrics, City Hospitals Sunderland NHS Foundation Trust, sunderland, UK

\subsection{6/archdischild-2018-rcpch.404}

From the coal face to strategic planning many assumptions are made about what staff do when working and the cost of training to trusts. It is assumed that less experienced staff earlier in their careers take longer to manage clinical problems than senior doctors. This assumption has been investigated in adult acute care settings. ${ }^{1}$ The present study was stage 1 of a quality improvement approach looking at factors that might detract from patient throughput.

One medical student (AF) observed individual patient assessments by medical staff over 4 weeks. Time spent undertaking different activities from point of first contact up to discharge were recorded. Post-discharge activity was recorded separately, plus qualitative data relating to outcomes.

43 consultations were observed. All assessments were completed within 4 hours.Systematic delays varied between patients but contributed to $19 \%$ of total time. Documentation made up $12 \%$ of patient contact time. A number of simple issues were identified as contributing to time delays - eg difficulties in communication between hospitals, incorrect labelling. Seniors were significantly quicker in assessing patients than junior trainees, and also spent the least time with the patients. Total time spent on patient care was not significantly different across all grades of juniors and consultants. Time spent on senior reviews was significantly greater for junior trainees compared to middle grades.

These findings are similar to observations in adult medicine about assessment activity. ${ }^{1}$ They provide some insights into the relative costs and utility of different grades of staff. Understanding how work occurs gives an opportunity to develop new working practices. Senior medical staff are more efficient in clinical assessment, but no more efficient in documentation than juniors. The next phase of this study is to work within the department to highlight opportunities for greater efficiency in relation to systematic delays and documentation.

\section{REFERENCE}

1. Sabin J, et al. 'The time it takes...' How doctors spend their time admitting a patient during the acute medical take. Clin Med (Lond) 2016 August; 16(4):320 4. 